\title{
La mortalidad por enfermedad diarreica en México: ¿problema de acceso o de calidad de atención?
}

Hortensia Reyes, M.C., M. en C., (1) Patricia Tomé, M.C., M. en C.,1) Gonzalo Gutiérrez, M.C., M.S.P., (2) Leticia Rodríguez, Enf. Sanit., ${ }^{(1)}$ Maribel O rozco, M.C., M. en C., ${ }^{(1)}$ Héctor Guiscafré, M.C., M. en C. ${ }^{(1)}$

\author{
Reyes $H$, Tomé $P$, Gutiérrez $G$, \\ Rodríguez L, Orozco M, Guiscafré $H$. \\ La mortalidad por enfermedad \\ diarreica en México: \\ ¿problema de acceso o de calidad de atención? \\ Salud Publica Mex 1998;40:316-323.
}

\section{Resumen}

Objetivo. Evaluar el proceso de atención y el acceso a servicios de salud en menores de cinco años fallecidos por enfermedad diarreica (ED), en cuatro estados de la República mexicana. Material y métodos Se realizó un estudio transversal que incluyó defunciones ocurridas durante un año; mediante autopsia verbal se obtuvo información de características clínicas, atención durante la enfermedad y acceso a los servicios de salud; se realizó un análisis descriptivo y comparativo de acuerdo con el tamaño de la localidad. Resultados. De 553 defunciones, la mayoría ocurrió en niños sin derecho a seguridad social o residentes en localidades rurales; la muerte en el hogar, la edad menor a un año y la corta evolución de la enfermedad caracterizaron a más de la mitad de los casos. Se proporcionó terapia de hidratación oral por decisión de la madre a casi $75 \%$ de los niños; $20 \%$ recibió atención no médica, principalmente de curanderos. La atención médica fue otorgada en $60 \%$ de los casos; a una elevada proporción le fue indicado algún medicamento y no se le envió a hospital ni se recomendó regresar ante la presencia de signos de alarma; la indicación de hidratación oral fue mayor por médicos de servicios públicos y en lo calidades rurales; en éstas, el acceso geo grafico y económico fue menor. Conclusiones. Si bien se confirmó el problema de acceso a los servicios de salud, se identificaron fallas importantes en la atención médica primaria. Se propone la creación de unidades docente-asistenciales como una estrategia para la reducción de la mortalidad por ED.

\author{
Reyes $H$, Tomé $P$, Gutiérrez $G$, \\ Rodríguez L, Orozco M, Guiscafré H. \\ Mortality due \\ to diarrhea in Mexico: \\ A problem of accessibility or quality of care? \\ Salud Publica Mex 1998;40:316-323.
}

\begin{abstract}
A bstract
Objective. To evaluate the access to health services and the process of care provided to children under five years old who died from acute diarrhea (AD). Material and methods. In four states of Mexico, a cross-sectional study was carried out. D eaths from AD that ocurred in the lapse of one year were included. Information about clinical characteristics of the illness, process of care and access to the health facilities were obtained through verbal autopsy. D escriptive analysis and comparison of data between rural and urban communities were performed. Results. Fivehundred and fifty three deaths were analysed. Most of the children were not enrolled in a public care health system. The main children's characteristics were the following:residence in rural communities; age less than one year; died at home or had short illness duration. Geographic and economic access barriers were identified in rural areas. 0 ral rehydration salts were provided as a part of household treatment in about $75 \%$ of children. $20 \%$ of them received non-medical care, specially from traditional healers. Primary medical care was provided to $60 \%$ of cases; the drawbacks of the process were related to a high rate of medication prescription, lack or referral to hospital, and lack of recommendation for a new visit in presence of alarm signs. Public physicians and those from rural communities prescribed oral rehydration therapy in a greater proportion. Conclusions. The problem of access to health services in rural communities was confirmed. However, low
\end{abstract}

(1) Unidad de Investigación Epidemiológica y en Servicios de Salud, Centro Médico N acional Siglo XXI. Coordinación de Investigación Médica. Instituto Mexicano del Seguro Social, México.

(2) Consejo N acional de Vacunación, Secretaría de Salud, México.

Fecha de recibido: 8 de enero de 1998 • Fecha de aprobado: 2 de abril de 1998

Solicitud de sobretiros: Dra. Hortensia Reyes. Unidad de Investigación Epidemiológica y en Servicios de Salud, Centro Médico Nacional Siglo XXI. Coordinación de Investigación Médica. Instituto Mexicano del Seguro Social. Av. Cuauhtémoc 330, colonia Doctores, 06725 México, D.F., México. 
Palabras clave: diarrea/mortalidad; calidad de la atención de salud; accesibilidad a los servicios de salud; México quality of care provided by primary level physicians was found in both rural and urban areas. We propose the creation of clinical teaching centers to improve the quality of the process of medical care as a strategy to decrease mortality due to $A D$.

Key words: diarrhea/mortality; quality of health care; health services accesibility; Mexico
$L$ a enfermedad diarreica (ED) ha sido una de las prioridades en los programas de salud en México a fin de disminuir la mortalidad en los menores de cinco años. Como resultado, en el lapso de 1990 a 1996 se observó una importante aceleración en la tendencia descendente de la mortalidad infantil y prescolar por este padecimiento y se logró una reducción de $69.4 \%{ }^{1}$ Dicha tendencia no fue homogénea y muestra dos etapas: la primera, que comprende hasta 1993 y en la que el descenso fue mayor (56\% en relación con 1990), derivado principalmente de un incremento en el uso de la terapia de hidratación oral (THO), de la mejoría de las condiciones sanitarias y de la intensificación de acciones para elevar la cobertura de vacunación antisarampionosa. ${ }^{2}$ En los años posteriores se observó una segunda etapa de descenso más lento, que probablemente estuvo relacionada con el efecto de consolidación de las acciones mencionadas.

No obstante los avances logrados, el número de muertes por ED sigue siendo injustificadamente elevado; en 1995 esta enfermedad fue la cuarta causa de mortalidad infantil con una tasa de 127 por 100000 nacidos vivos registrados, y la tercera en mortalidad prescolar (tasa de 15 por 100000 niños de este grupo de edad). El problema fue más evidente en algunos estados del país con condiciones de pobreza extrema, como Chiapas y Oaxaca. ${ }^{3}$

Esas cifras sugieren la existencia de problemas adicionales que favorecen la persistencia de defunciones prevenibles y que es posible que estén relacionados con deficiencias en el proceso de atención de los niños que fallecen por ED. Sin embargo, la identificación de este proceso es difícil, sobre todo en áreas rurales donde una elevada proporción de los niños muere en su hogar. ${ }^{4}$ El certificado de defunción, que brinda los datos básicos para la información estadística de la que se desprenden muchas de las políticas de salud, no incluye la información necesaria para poder hacer un análisis más amplio, y se ha demostrado que con mucha frecuencia existen errores en ese documento, lo cual impide una interpretación confiable.5,6
Una alternativa que ha resultado útil para evaluar el proceso de la atención es la autopsia verbal (AV), que por medio de una entrevista a los familiares de la persona fallecida permite obtener información acerca de la enfermedad terminal. ${ }^{7-8}$ Es por ello que el objetivo del presente trabajo fue analizar, por medio de la $\mathrm{AV}$, las características de dicho proceso en muertes por ED en menores de cinco años en cuatro estados del país, prioritarios para los programas de salud debido a sus condiciones de rezago extremo, ${ }^{9}$ con el fin de establecer un diagnóstico situacional y evaluar la necesidad de estrategias particulares para la prevención de muertes por ED en niños.

\section{Material y métodos}

Se llevó a cabo un estudio transversal en los estados de Chiapas, Guerrero, Hidalgo y Oaxaca, durante 1994. Se revisaron los certificados de defunción de muertes ocurridas entre el 1 de septiembre de 1993 y el 30 de septiembre de 1994, concentrados en el Departamento de Estadística de la Unidad de Planeación perteneciente a la Secretaría de Salud (SSA) de cada estado. Se incluyeron defunciones de niños menores de cinco años ocurridas en ese periodo, con diagnóstico de muerte por enfermedad diarreica o deshidratación en el certificado de defunción (causa básica o secundaria) y confirmada por AV. Debido al elevado número de defunciones, para Oaxaca y Chiapas se calculó una muestra que permitiera una precisión de 0.05 con un nivel de confianza de $95 \%$; se ponderó la muestra de acuerdo con la proporción de defunciones por jurisdicción sanitaria (región administrativa de la SSA) y la selección se efectuó mediante un procedimiento aleatorio en cada jurisdicción. En los estados de Guerrero e Hidalgo se analizó el total de las defunciones ocurridas en el periodo de estudio.

Una enfermera coordinadora de cada estado se encargó de seleccionar los certificados trimestralmente. Con fines logísticos cada estado fue dividido en zonas geográficas, asignando a cada zona un encuestador; 
este personal estuvo constituido por enfermeras sanitarias y trabajadores de salud comunitarios de la SSA previamente capacitados en la técnica de $\mathrm{AV}$ y en su mayoría residentes de la misma zona, lo que permitió la comunicación en lengua indígena cuando fue necesario. A ellos se les entregó una copia de los certificados de defunción seleccionados para su zona con la finalidad de localizar a la familia y efectuar la visita domiciliaria. En esa visita se realizó la AV a la madre o responsable del niño durante su última enfermedad, a fin de obtener las variables de estudio: edad y sexo del niño, sitio de la defunción, derechohabiencia a instituciones de seguridad social, tamaño de la localidad de residencia y características del cuadro clínico; se registraron detalladamente el tipo y las características de la atención recibida en el hogar y por personal de salud no médico y médico relacionadas con indicación de medicamentos, hidratación oral, modificaciones en la alimentación y envío a algún hospital; asimismo, se interrogó sobre las condiciones de acceso a los servicios de salud.

Uno de los investigadores, revisó todas las AV, calificando como causa de muerte por enfermedad diarreica cuando, por medio de la entrevista, se identificara un padecimiento caracterizado por más de tres evacuaciones en 24 horas, disminuidas de consistencia y diferentes al patrón normal de evacuaciones del niño, con alguna de las siguientes complicaciones: a) deshidratación: por lo menos dos de los siguientes datos: inquieto o irritable, ojos hundidos o llanto sin lágrimas, fontanela deprimida, sequedad de boca o saliva espesa, sed, respiración profunda y letargo; b) septicemia: fiebre o hipotermia en menores de un mes con ataque al estado general, sin datos de deshidratación; c) complicación abdominal: distensión abdominal persistente (por más de 12 horas), que no cediera al hidratar al paciente o que no se asociara a deshidratación.

Para garantizar la calidad de la información, la coordinadora de cada estado realizó, junto con cada encuestador, $10 \%$ de las entrevistas y, en los casos de información dudosa, efectuó una nueva entrevista. Además, uno de los investigadores llevó a cabo la coordinación y la supervisión permanente en cada estado, garantizando así la uniformidad de los procedimientos. Con el fin de evitar sesgo de memoria y siguiendo las recomendaciones al respecto, ${ }^{10}$ todas las $\mathrm{AV}$ se efectuaron en los siguientes seis meses posteriores a la defunción.

Se realizó un análisis estadístico descriptivo de las variables de estudio y la prueba $\chi^{2}$ para la comparación de algunas variables de interés entre defunciones ocurridas en localidades rurales (menores de 2500 habi- tantes) y urbanas y entre el proceso de atención otorgado por médicos privados e institucionales.

\section{Resultados}

Se seleccionaron 808 defunciones que reunieron el criterio establecido, de las que se eliminaron 99 por la imposibilidad de localizar a las familias de los fallecidos. De las 709 AV que se hicieron, en 156 (22\%) no se confirmó enfermedad diarreica como causa de muerte, de tal manera que el análisis se realizó sobre 553 casos: 98 de Chiapas, 171 de Guerrero, 110 de Hidalgo y 174 de Oaxaca. Debido a la similitud de los resultados en los cuatro estados, los datos se agruparon para su presentación.

Las características de las defunciones se muestran en el cuadro I, en el que se destaca lo siguiente: poco más de la mitad de los niños tenían menos de un año de edad; las dos terceras partes de las defunciones ocurrieron en el hogar; casi ningún niño era derechohabiente de alguna institución y más de la mitad habitaba localidades rurales. Más de $80 \%$ de los casos fueron atendidos por personal de salud, la mayoría por médicos. La evolución de la enfermedad fue menor a tres días en más de la mitad de los niños y los signos de alarma identificados con más frecuencia fueron: vómito, boca seca, sed y fiebre.

El manejo que se hizo del enfermo en el domicilio antes de solicitar alguna atención se caracterizó por una baja proporción de administración de antibióticos sin indicación médica (13.4\%); $73.8 \%$ de los niños recibieron terapia de hidratación oral (THO) y, aunque a más de la mitad de los niños se les ofrecieron líquidos en mayor cantidad que la habitual, sólo una tercera parte recibieron sales de hidratación oral (SHO); a $15.5 \%$ de los niños que se alimentaban al seno materno éste les fue suspendido y a menos de $10 \%$ se les restringieron los alimentos o se les puso en ayuno. $\mathrm{Al}$ analizar el manejo en el hogar de 95 casos que no fueron atendidos por personal de salud destacó la elevada proporción de niños que recibieron THO (89.3\%) y SHO (51.1\%); los motivos que refirieron las madres para no haber solicitado atención fueron, principalmente, la falta de dinero para llegar a un servicio de salud $(34 \%)$, no considerarlo necesario $(21.3 \%)$ y la lejanía de los servicios (14.9\%).

En el cuadro II se muestra información acerca de la atención prestada por personal no médico, que fue otorgada en 121 casos (21.9\%). Casi la mitad (47\%) correspondió a curanderos y $34.7 \%$ fueron promotores de salud; el $18 \%$ restante se distribuyó entre boticarios y enfermeras. Resalta que más de $60 \%$ de los niños aten- 
Cuadro I

Características de la población estudiada en RELACIÓN CON ENFERMEDAD DIARREICA. MÉXICO, 1993-1994

Variable

$$
\begin{array}{ll}
n=553 \\
\hline n \quad \%
\end{array}
$$

\begin{tabular}{lll} 
Sexo & & \\
Masculino & 295 & 53.3 \\
\hline Femenino & 258 & 46.7
\end{tabular}

Edad

\begin{tabular}{lll}
$<6$ meses & 151 & 27.3 \\
\hline $6-11$ meses & 172 & 31.1 \\
\hline $1-4$ años & 230 & 41.6
\end{tabular}

Sitio de atención

\begin{tabular}{lrr} 
Sólo hogar & 95 & 17.2 \\
\hline Personal no médico & 121 & 21.8 \\
\hline Personal médico & 337 & 60.9
\end{tabular}

Sitio de defunción

\begin{tabular}{lrr} 
Hogar & 340 & 61.5 \\
\hline Unidad de 1er. nivel & 31 & 5.6 \\
\hline Unidad de 2do. nivel & 105 & 19.0 \\
\hline Traslado & 77 & 13.9
\end{tabular}

Sin derechohabiencia institucional

Tamaño de localidad

\begin{tabular}{lll}
$<500$ habitantes & 129 & 23.3 \\
\hline $500-2499$ habitantes & 213 & 38.5 \\
$>2499$ habitantes & 211 & 38.2
\end{tabular}

Días de evolución

\begin{tabular}{lrl}
$<24$ horas & 84 & 15.2 \\
\hline $1-3$ días & 258 & 46.7 \\
\hline 3 días & 211 & 38.1
\end{tabular}

Evacuaciones en 24 horas

\begin{tabular}{lrr} 
Mediana & 7 & -- \\
\hline Intervalo & $1-50$ & --
\end{tabular}

Signos de alarma

\begin{tabular}{lrr} 
Fiebre & 351 & 63.5 \\
\hline Vómito & 446 & 80.7 \\
\hline Sangre en evacuaciones & 79 & 14.3 \\
\hline O jos hundidos & 397 & 71.8 \\
\hline Boca seca & 435 & 78.7 \\
\hline Sed & 413 & 74.7 \\
\hline Fontanela hundida & 171 & 30.9 \\
\hline Respiración rápida & 327 & 59.1
\end{tabular}

didos por este tipo de personal recibieron dos o más consultas; a pesar de que la proporción de envíos a hospital fue muy baja, la indicación de hidratación oral

\section{Cuadro II \\ Atención otorgada por Personal no médico* en RELACIÓN CON ENFERMEDAD DIARREICA. MÉxıCo, 1993-1994}

$$
n=121
$$

$N$ úmero de atenciones

\begin{tabular}{lll} 
Una & 39 & 32.2 \\
\hline Más de una & 82 & 67.7
\end{tabular}

Indicación (primera atención)

\begin{tabular}{lrr} 
Envío al hospital & 10 & 8.2 \\
\hline Medicamentos & 50 & 41.3 \\
\hline Antibióticos & 10 & 8.2 \\
\hline Sales de hidratación horal & 51 & 42.1 \\
\hline Suero comercial & 16 & 13.2 \\
\hline O tros líquidos & 41 & 33.8 \\
\hline Cualquier modificación de la alimentación & 11 & 9.1 \\
\hline Indicaciones de regresar con signos de alarma & 29 & 23.9 \\
\hline Suspensión de seno materno $\mathrm{n}=271^{\ddagger}$ & 7 & 2.6 \\
\hline Ayuno $\mathrm{n}=373^{\S}$ & 4 & 1.1 \\
\hline Restricción de alimentos $\mathrm{n}=373^{\S}$ & 3 & 0.8 \\
\hline
\end{tabular}

* Curandero $(n=57)$; promotor de salud $(n=41)$; enfermera y boticario $(n=23)$

₹ $N$ iños que al inicio de la enfermedad se alimentaban al seno materno

${ }^{\S} \mathrm{N}$ iños que al inicio de la enfermedad ingerían otros alimentos

fue frecuente y hubo pocas indicaciones de antibióticos y modificaciones en la alimentación, aunque casi la mitad de los casos recibieron algún medicamento.

En contraste, en 337 casos (60.9\%) se proporcionó alguna atención médica, principalmente por parte de médicos privados. En el cuadro III se presentan las características de la primera consulta y se comparan las indicaciones entre médicos privados e institucionales (pertenecientes en su mayoría a la SSA o al Instituto Mexicano del Seguro Social). Puede observarse que, en general, la atención otorgada por los médicos que trabajan en servicios públicos fue mejor, sobre todo en las indicaciones de $\mathrm{SHO}$ y el menor uso de antibióticos; los médicos privados se caracterizaron por indicar con mayor frecuencia el suero comercial y, además, por hacer mayores modificaciones en la alimentación que los médicos institucionales. Sin embargo, la indicación de medicamentos fue sumamente elevada en ambos grupos y no hubo diferencia con los médicos privados en las recomendaciones a las madres para la identificación de signos de alarma; en ambos grupos de médicos la frecuencia de envío a hospital fue baja. La mediana del tiempo tanscurrido entre el inicio de la enfermedad y la primera atención por parte de personal de salud fue de 20 horas. 


\section{Cuadro III \\ Atención otorgada por el Personal médico en RELACIÓN CON ENFERMEDAD DIARREICA. MÉxıco, 1993-1994}

\begin{tabular}{|c|c|c|}
\hline & $\begin{array}{l}\text { Médico } \\
\text { privado } \\
n=211 \\
\%\end{array}$ & $\begin{array}{c}\text { Médico de } \\
\text { alguna institución } \\
n=126 \\
\%\end{array}$ \\
\hline \multicolumn{3}{|l|}{$N$ úmero de atenciones } \\
\hline Una & 34.1 & 34.9 \\
\hline Más de una & 65.8 & 65.0 \\
\hline \multicolumn{3}{|l|}{ Indicación (primera consulta) } \\
\hline Envío al hospital & 7.5 & $15.0 *$ \\
\hline Medicamentos & 93.3 & $80.9 \ddagger$ \\
\hline Antibióticos & 28.4 & $11.9 \ddagger$ \\
\hline Sales de hidratación oral & 36.9 & $76.9 \ddagger$ \\
\hline Suero comercial & 38.8 & $8.7 \ddagger$ \\
\hline 0 tros líquidos & 32.2 & 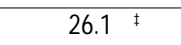 \\
\hline $\begin{array}{l}\text { Cualquier modificación } \\
\text { de la alimentación }\end{array}$ & 22.7 & $11.1 \S$ \\
\hline \multicolumn{3}{|l|}{ Indicaciones de regresar } \\
\hline con signos de alarma & 43.6 & 36.5 \\
\hline \multicolumn{3}{|l|}{ Suspensión de seno } \\
\hline materno $n=271^{\#}$ & 7.4 & $2.9 *$ \\
\hline Ayuno $n=373^{f}$ & 7.6 & $0.5 \S$ \\
\hline $\begin{array}{l}\text { Restricción } \\
\text { de alimentos } n=373^{\S}\end{array}$ & Restricción & $2.4 \S$ \\
\hline \multicolumn{3}{|c|}{$\begin{array}{l}* p<0.05 \\
* N \text { iños que al inicio de la enfermedad se alimentaban al seno materno } \\
{ }^{f} \mathrm{~N} \text { iños que al inicio de la enfermedad ingerían otros alimentos } \\
{ }_{f} p<0.001\end{array}$} \\
\hline
\end{tabular}

En el cuadro IV se presenta la información de acceso según el tamaño de la localidad. Se observa que las defunciones en el hogar fueron más frecuentes en localidades rurales, sobre todo en niños sin derecho a seguridad social. Es evidente la dificultad que existe para poder tener acceso a los servicios de salud en las localidades menores de 500 habitantes, ya que es necesaria más de una hora de transporte al servicio de salud más cercano en más de la mitad de los casos estudiados; las barreras económicas, aunque en baja proporción, también se refirieron como motivos de no hospitalización.

El cuadro $V$ proporciona datos acerca del proceso de atención médica y por personal no médico, analizado también de acuerdo con el número de habitantes de las localidades. No se encontró diferencia en la proporción de casos que recibieron atención por personal de salud no médico, que se esperaría mayor en las comunidades más pequeñas. En contraste, destaca la

\section{Cuadro IV \\ ACCESIBILIDAd POR TAMAÑo DE LOCALIDAD. Atención de la enfermedad diarreica en México, 1993-1994}

\begin{tabular}{lccc} 
& \multicolumn{3}{c}{$\begin{array}{c}\text { Tamaño de localidad } \\
\text { (No. de habitantes)* }\end{array}$} \\
\cline { 2 - 4 } & $\begin{array}{c}<00 \\
\mathrm{n}=129\end{array}$ & $\begin{array}{c}500-2 \\
\mathrm{n}=213\end{array}$ & $\mathrm{n}=211$ \\
& & & \\
Defunción en el hogar & 65.9 & 68.1 & $52.1^{\ddagger}$ \\
\hline Sin seguridad social & 94.6 & 93.4 & $86.7^{\S}$
\end{tabular}

Tiempo de recorrido al servicio

de salud más cercano (minutos)

\begin{tabular}{llll}
$<30$ & 20.2 & 45.5 & 63.5 \\
\hline $30-60$ & 18.6 & 16.9 & 20.9 \\
\hline$>60$ & 61.2 & 37.6 & $15.6^{\ddagger}$
\end{tabular}

Motivos de no hospitalización $\quad n=121^{\#} \quad n=194^{\#} \quad n=187^{\#}$

$\begin{array}{llll}\text { El médico no lo envió } & 31.4 & 48.9 & 41.7\end{array}$

\begin{tabular}{lrrc}
\hline La madre no lo & & & \\
consideró necesario & 16.5 & 18.5 & 18.1 \\
\hline Estaba muy lejos & 17.3 & 13.9 & $3.7^{\ddagger}$ \\
\hline N o tenía dinero para el traslado & 10.7 & 16.9 & $9.7^{f}$ \\
\hline O tros & 24.1 & 1.8 & 26.8
\end{tabular}

\# En niños que no fueron hospitalizados

$*$ Diferencias entre localidades $<2500$ habitantes y $>2499$ habitantes

$\ddagger p<0.001$

${ }^{\S} p<0.005$

f $p<0.05$

mayor proporción de utilización de servicios de salud a expensas de atención médica, que se incrementó en las localidades urbanas: $53.8 \%$ de los casos en comunidades menores de 2500 habitantes $v s 72.5 \%$ en comunidades mayores. Es interesante observar que la indicación de medicamentos, incluyendo antibióticos y sueros comerciales, fue similar entre los médicos, independientemente del tamaño de la localidad, así como también la frecuencia de recomendaciones a la madre para regresar a consulta ante la presencia de signos de alarma, que fue en menos de la mitad de las consultas. Llama la atención que la única diferencia se observó en la frecuencia de la prescripción de SHO tanto por personal no médico como por los médicos, la cual fue mayor en consultas otorgadas en las localidades más pequeñas.

\section{Discusión}

La mortalidad por ED en niños ha sido motivo de numerosos estudios en todo el mundo, lo que refleja la necesidad de contar con mejor información para sus- 


\section{Cuadro V}

Proceso de atención de la enfermedad diarreica SEGÚN TAMAÑo DE LOCALIDAD. MÉXICO, 1993-1994

\begin{tabular}{|c|c|c|c|}
\hline & \multicolumn{3}{|c|}{$\begin{array}{l}\text { Tamaño de localidad* } \\
\text { (No. de habitantes) }\end{array}$} \\
\hline & $\begin{array}{l}<500 \\
n=129\end{array}$ & $\begin{array}{c}500-2499 \\
n=213\end{array}$ & $\begin{array}{l}>2499 \\
n=211\end{array}$ \\
\hline \multicolumn{4}{|l|}{ Tratamiento indicación ${ }^{\ddagger}$} \\
\hline Personal no médico & $n=34$ & $n=49$ & $n=38$ \\
\hline Sales de hidratación oral & 47.1 & 55.1 & $21.1^{\S}$ \\
\hline Modificaciones en la alimentación & 17.6 & 8.2 & 5.3 \\
\hline Envío a hospitalización & 5.9 & 12.2 & 7.9 \\
\hline Personal médico & $n=68$ & $n=116$ & $n=153$ \\
\hline Medicamentos & 94.1 & 87.1 & 87.6 \\
\hline Antibióticos & 22.1 & 16.4 & 24.2 \\
\hline Sales de hidratación oral & 63.2 & 55.2 & $44.4^{\S}$ \\
\hline Suero comercial & 23.5 & 25.0 & 31.4 \\
\hline 0 tros líquidos & 30.9 & 33.6 & 26.8 \\
\hline Modificaciones en la alimentación & 17.6 & 17.2 & 18.9 \\
\hline Envío a hospital & 8.8 & 9.5 & 11.8 \\
\hline \multicolumn{4}{|l|}{ Indicaciones de regresar } \\
\hline con signos de alarma & 48.5 & 36.2 & 41.2 \\
\hline $\begin{array}{l}* \text { Diferencias entre localidades }<2500 \text { y } \\
\text { F Indicación dada en la primera consulta } \\
\text { s } p<0.01\end{array}$ & $\begin{array}{l}y>24 \\
a\end{array}$ & & \\
\hline
\end{tabular}

tentar científicamente los programas de atención relacionados con este problema de salud. Los resultados de este trabajo permiten un acercamiento a las condiciones que se presentaron durante la enfermedad que llevó a la muerte a niños con ED, así como a las conductas de los responsables de la atención de estos niños, en regiones de México donde persiste una elevada mortalidad por este padecimiento.

En primer lugar, es notoria la baja proporción de defunciones que se presentaron en niños pequeños, lo que contrasta con las cifras nacionales de mortalidad para 1995 en las que más de $80 \%$ de las defunciones en menores de cinco años corresponde a niños de menos de un año de edad. ${ }^{3}$ Estos datos sugieren que hay subregistro de muerte en niños de pocos meses de edad. El subregistro ya ha sido documentado por informes derivados de estimaciones indirectas ${ }^{11} \mathrm{y}$ se ha considerado que la subestimación de la mortalidad infantil en algunos estados como Chiapas y Oaxaca puede ser superior a $50 \%$ y que en el estado de Guerrero llega a $80 \%$; recientemente, este grupo de trabajo ha encontrado un subregistro de $69 \%$ en localidades rurales de Guerrero, principalmente debido a ED e infecciones respiratorias agudas. ${ }^{12}$
En este estudio, el análisis del proceso de atención que se brindó en el hogar muestra que, en general, fue apropiado. Asimismo, la solicitud temprana de atención médica en la mayor parte de los casos pudiera ser consecuencia de la identificación de signos de alarma o datos de deshidratación; en un trabajo previo realizado en localidades con buen acceso a servicios de salud se encontró que las madres de niños con ED no identificaron signos de alarma para decidirse a solicitar la atención. ${ }^{13}$ Es probable que los resultados de este estudio reflejen el impacto de las estrategias de capacitación a la población, que se han efectuado con mayor énfasis en zonas de alto riesgo como las que estuvieron representadas en esta muestra. Es necesario reconocer, sin embargo, que la frecuencia de uso de SHO por iniciativa de las madres fue insatisfactoria y que esa situación parece no ser exclusiva de los casos que fallecen, como lo demostraron los resultados de la Encuesta sobre el Manejo Efectivo de Casos de Diarrea en el Hogar (EMECADI 1993) en una muestra representativa para todo el país, donde la tasa de uso de SHO fue similar a la encontrada en este estudio. ${ }^{14}$ Por otro lado, es preocupante que casi uno de cada cinco niños haya fallecido sin haber recibido atención del personal de sauld y, aunque los principales motivos estuvieron relacionados con la falta de acceso geográfico o económico, se encontraron 20 casos de niños que fallecieron sin que se solicitara atención médica debido a que la madre no lo consideró necesario. Estos hallazgos muestran que aún es necesario consolidar la educación dirigida a las madres, con un enfoque amplio que aborde el papel de la familia como responsable de la provisión de salud en el hogar.

Un aspecto particularmente relevante lo constituye la información relacionada con la atención recibida por el personal de salud. La solicitud de atención no médica en uno de cada cuatro niños refleja la importancia de este personal de salud en las comunidades estudiadas, sobre todo el papel del curandero quien atendió en casi la mitad de las ocasiones; al contrario de lo esperado, los boticarios tuvieron poca participación en la atención, lo que coincide con lo notificado en un estudio efectuado en una muestra de población urbana en la Ciudad de México. ${ }^{15}$ Los errores identificados en este personal, principalmente en la prevención o tratamiento de deshidratación, evidencian la necesidad de insistir en su incorporación a los programas de capacitación.

La comparación de la atención otorgada entre médicos institucionales y privados, permite identificar información interesante que merece un comentario. Destaca la diferencia en la prescripción de hidratación 
oral, con una marcada preferencia de los médicos privados por indicar el consumo de sueros comerciales, en contraste con la elevada proporción de indicación de SHO por parte de los médicos que trabajan en instituciones de salud. Por otro lado, se considera alarmante la baja frecuencia de envíos al hospital y de consultas en las que se dieron indicaciones para regresar en caso de signos de alarma. A pesar de que el análisis presentado corresponde a la primera consulta, las fallas en el proceso de atención médica en primer nivel se manifiestan por la menor cantidad de defunciones hospitalarias en niños con cuadros de corta evolución, en quienes no se identificó la necesidad de atención especializada, ya sea por gravedad en el momento de la consulta o por el riesgo de complicación.

La falta de acceso a los servicios médicos es un problema que puede influir considerablemente en la persistencia de niveles de mortalidad elevados, de tal manera que se seleccionaron algunos indicadores que permitieran explorar más a fondo este aspecto, y se analizaron de acuerdo con la ruralidad de las localidades. La elevada proporción de defunciones ocurridas en el hogar es un indicador que no ha logrado modificarse a pesar de la reducción absoluta de las muertes en niños en los últimos años, aun en sitios con acceso adecuado a los servicios de salud. ${ }^{2}$ En los estados estudiados, los impedimentos geográficos para tener acceso a la atención médica y el no tener derecho a los servicios de las instituciones de seguridad social fueron, efectivamente, mayores en localidades rurales, lo que destaca la necesidad de capacitar aún más al personal de salud comunitario de esas áreas para lograr un adecuado manejo de la ED. Sin embargo, cabe hacer notar que más de la mitad de los niños que fallecieron aun en comunidades muy pequeñas recibieron atención médica durante su enfermedad y pocos casos fueron referidos a un hospital, lo que posiblemente favoreció las defunciones en el domicilio; en esos casos se identifica el problema de falta de continuidad en la atención, derivada de deficiencia en los servicios. El hecho de que el principal motivo de que el enfermo no haya sido hospitalizado fuera la falta de indicación del médico y no las barreras geográficas o los impedimentos económicos, sustenta esa afirmación.

Por otro lado, si bien en las localidades urbanas hubo más casos de solicitud de atención médica, los errores en el proceso de atención se presentaron independientemente del tamaño de la localidad. Además, en los médicos de las áreas urbanas la prescripción de SHO fue significativamente más baja, lo que probablemente se deba a la influencia de otros factores como la presencia de la propaganda de los laboratorios priva- dos que favorece la indicación de sueros comerciales, tal como se ha establecido en estudios previos. ${ }^{16,17}$

En conclusión, el análisis de este trabajo muestra que, si bien persiste el problema de acceso a los servicios de salud, también existen fallas importantes en los mismos, de manera especialmente grave en lo que corresponde a los médicos de atención primaria. Es preocupante observar que en regiones como las estudiadas, a pesar de las barreras económicas, geográficas y culturales que crean condiciones particularmente difíciles, la población solicita atención, pero muchas veces el tratamiento indicado por los médicos no es el apropiado; sin duda, este aspecto es relevante, ya que se ha demostrado que los errores en el proceso de la atención médica son un factor determinante para el mal pronóstico y el elevado número de muertes infantiles por ED. ${ }^{18}$

Los aciertos en los programas nacionales que han resultado en la disminución de la mortalidad en los últimos años son incuestionables; el impacto en la población se refleja en una mejor información de la ED, un aumento del uso de la THO en el hogar y mayor oportunidad en la solicitud de atención; a la fecha se ha superado la meta propuesta al inicio de la presente década para la reducción de la mortalidad por ED en niños hacia el año 2000. La experiencia que se ha obtenido a partir del programa nacional para el control de estos padecimientos ha permitido desarrollar otras estrategias para la atención del niño, con lo que se pretende alcanzar una reducción de $85 \%$ para el fin del siglo. ${ }^{1}$ No obstante, se considera que para lograr esos resultados es necesario tener un nuevo abordaje del problema; es indiscutible que deberán continuarse las acciones implantadas, pero tendrán que establecerse de manera prioritaria nuevas acciones para mejorar la calidad de la atención médica, ya que las deficiencias en este aspecto han persistido. La capacitación a gran escala que se ha llevado a cabo hasta ahora no ha bastado para corregir los errores médicos que inciden en el elevado número de muertes infantiles que pueden prevenirse. El Programa de Atención a la Salud del Niño, en el cual se aborda de manera integral el cuidado del menor de cinco años, propone la creación de unidades docente-asistenciales que brinden una atención de excelencia a niños con ED y se capacite simultáneamente a los médicos dentro del mismo servicio; es recomendable el análisis de las muertes evitables, con información obtenida por medio de la realización rutinaria de $\mathrm{AV}$ en defunciones de casos de las propias unidades. Esos centros también son los sitios ideales para enseñar a las madres de familia el manejo adecuado del niño con ED en el hogar y para 
solicitar la atención con oportunidad. Es necesario que en esas unidades se incorpore permanentemente la capacitación a los médicos privados como una prioridad, así como a los estudiantes de medicina durante su aprendizaje clínico de pregrado. El tratamiento adecuado de los niños con ED, ciertamente será uno de los elementos fundamentales para reducir las cifras de mortalidad por ese padecimiento.

\section{Agradecimientos}

Este trabajo se realizó con el apoyo financiero del Programa Solidaridad SSA, mediante acuerdo de colaboración con el Banco Mundial. Se agradece la participación del personal de la SSA en los estados de Chiapas, Guerrero, Hidalgo y Oaxaca en los aspectos logísticos del trabajo de campo.

\section{Referencias}

1. Consejo Nacional de Vacunación. Programa de Atención a la Salud del N iño. México, D.F.: Secretaría de Salud, 1997.

2. Consejo $\mathrm{N}$ acional para el Control de las Enfermedades Diarreicas (CO N ACED). Programa N acional de Control de las Enfermedades Diarreicas 1993-1994. México, D.F.: Sistema N acional de Salud, 1993.

3. Dirección General de Estadística e Informática. Subsecretaría de Planeación. Mortalidad 1995. México, D.F.: Secretaría de Salud, 1995.

4. Villa S, Guiscafré H, Martínez H, Urbán JC, Reyes S, Lezana MA et al. Muertes en el hogar en niños con diarrea o infección respiratoria aguda después de haber recibido atención médica. Bol Med Hosp Infant Mex 1994;51:233-242.

5. A nónimo.The quality of cause-of-death statistics. Editorial. Am J Public Health 1987;77:137-139.
6. C revena-Horney P, Real-MataT. Integridad de las fuentes de defunción y problemas de la transcripción de la información en ocho estados de la República Mexicana. Salud Publica Mex 1989;31:591-600.

7. Martínez $H$, Reyes $H$, Tomé $P$, G uiscafré $H$, Gutiérrez $G$. La autopsia verbal: una herramienta para el estudio de la mortalidad en niños. Bol Med Hosp Infant Mex 1993;50:57-63.

8. Reyes $H$, Tomé $P$, G uiscafré $H$, Romero G, Portillo E, Rodríguez R et al. Autopsia verbal en niños con infección respiratoria y diarrea aguda. Análisis del proceso enfermedad-atención-muerte. Bol Med Hosp Infant Mex 1993:50:7-16.

9. Lozano R. El peso de la enfermedad en México: avances y desafíos. En: Frenk J, ed. $O$ bservatorio de la salud. $N$ ecesidades, servicios, políticas. México, D.F.: Fundación Mexicana para la Salud, 1997:23-61.

10. G ray R.Verbal autopsy: U sing interviews to determine causes of death in children. Baltimore: Institute for International Programs, The Johns Hopkins University, 0 ccasional Paper Series, N 0. 14, 1991.

11. Gómez-De León J, PartidaV. N iveles de mortalidad infantil y fecundidad en México, por entidad federativa, 1990. Rev Med Soc 1994;2: 97-135.

12. Tomé P, Reyes H, Piña C, Rodríguez L, Gutiérrez G. Características asociadas al subregistro de muerte en niños del estado de Guerrero, México. Salud Publica Mex 1997;39:523-529.

13. Pérez-C uevas R, Guiscafré $H$, Romero G, Rodríguez L, Gutiérrez G. Mothers' health-seeking behaviour in acute diarrhoea in Tlaxcala, Mexico. J Diarrhoeal Dis Res 1996;14:260-268.

14. Dirección General de Epidemiología. Subsecretaría de Coordinación y Desarrollo. Encuesta sobre el Manejo Efectivo de Casos de Diarrea en el Hogar 1993. México, D.F.: Secretaría de Salud, 1994.

15. Calva JJ, Cerón E, Bojalil R, Holbrook A. Uso de antibióticos en una comunidad de la Ciudad de México. Il. Encuesta de compras en farmacias. Bol Med Hosp Infant Mex 1993;50:145-150.

16. Soumerai SB. Factors influencing prescribing. Aust J Hosp Pharm 1988;3:9-15.

17. Pérez-C uevas $R$, Muñoz 0 , Guiscafré $H$, Reyes $H$, Tomé $P$, Gutiérrez $G$. Patrones de prescripción terapéutica en diarrea e infecciones respiratorias agudas en dos instituciones de salud. IV. Características de la prescripción médica. Gac Med Mex 1992;128:531-541.

18. Tomé P, Reyes H, Rodríguez L, Guiscafré H, Gutiérrez G. Muerte por diarrea aguda en niños: un estudio de factores pronósticos. Salud Publica Mex 1996;38:227-235. 